\title{
Learning Word Meaning and Grammatical Constructions from Narrated Video Events
}

\author{
Peter Ford Dominey \\ Institut des Sciences Cognitives, \\ CNRS \\ 67 Blvd. Pinel, \\ 69675 Bron Cedex, France \\ dominey@ isc.cnrs.fr
}

\begin{abstract}
The objective of this research is to develop a system for miniature language learning based on a minimum of prewired language-specific functionality, that is compatible with observations of perceptual and language capabilities in human development. In the proposed system, meaning is extracted from video images based on detection of physical contact and its parameters. Mapping of sentence form to meaning is performed by learning grammatical constructions that are retrieved from a construction inventory based on the constellation of closed class items uniquely identifying the target sentence structure. The resulting system displays robust acquisition behavior that reproduces certain observations from developmental studies, with very modest "innate" language specificity.
\end{abstract}

\section{Introduction}

Feldman et al. (1990) posed the problem of "miniature" language acquisition based on <sentence, image> pairs as a "touchstone" for cognitive science. In this task, an artificial system is confronted with a reduced version of the problem of language acquisition faced by the child, that involves both the extraction of meaning from the image, and the mapping of the paired sentence onto this meaning.

\section{Extraction of Meaning}

In this developmental context, Mandler (1999) suggested that the infant begins to construct meaning from the scene based on the extraction of perceptual primitives. From simple representations such as contact, support, attachment (Talmy 1988) the infant could construct progressively more elaborate representations of visuospatial meaning. Thus, the physical event "collision" is a form of the perceptual primitive "contact". Kotovsky \& Baillargeon (1998) observed that at 6 months, infants demonstrate sensitivity to the parameters of objects involved in a collision, and the resulting effect on the

\author{
Thomas Voegtlin \\ Institute for Theoretical Biology \\ Humboldt-Universität \\ Invalidenstraße 43 \\ D-10115 Berlin, Germany \\ Voegtlin@isc.cnrs.fr
}

collision, suggesting indeed that infants can represent contact as an event predicate with agent and patient arguments.

Siskind (2001) has demonstrated that force dynamic primitives of contact, support, attachment can be extracted from video event sequences and used to recognize events including pick-up, put-down, and stack based on their characterization in an event logic. The use of these intermediate representations renders the system robust to variability in motion and view parameters. Most importantly, Siskind demonstrated that the lexical semantics for a number of verbs could be established by automatic image processing.

\section{Sentence to meaning mapping:}

Once meaning is extracted from the scene, the significant problem of mapping sentences to meanings remains. The nativist perspective on this problem holds that the <sentence, meaning> data to which the child is exposed is highly indeterminate, and underspecifies the mapping to be learned. This "poverty of the stimulus" is a central argument for the existence of a genetically specified universal grammar, such that language acquisition consists of configuring the UG for the appropriate target language (Chomsky 1995). In this framework, once a given parameter is set, its use should apply to new constructions in a generalized, generative manner.

An alternative functionalist perspective holds that learning plays a much more central role in language acquisition. The infant develops an inventory of grammatical constructions as mappings from form to meaning (Goldberg 1995). These constructions are initially rather fixed and specific, and later become generalized into a more abstract compositional form employed by the adult (Tomasello 1999). In this context, construction of the relation between perceptual and 
cognitive representations and grammatical form plays a central role in learning language (e.g. Feldman et al. 1990, 1996; Langacker 1991; Mandler 1999; Talmy 1998).

These issues of learnability and innateness have provided a rich motivation for simulation studies that have taken a number of different forms. Elman (1990) demonstrated that recurrent networks are sensitive to predictable structure in grammatical sequences. Subsequent studies of grammar induction demonstrate how syntactic structure can be recovered from sentences (e.g. Stolcke \& Omohundro 1994). From the "grounding of language in meaning" perspective (e.g. Feldman et al. 1990, 1996; Langacker 1991; Goldberg 1995) Chang \& Maia (2001) exploited the relations between action representation and simple verb frames in a construction grammar approach. In effort to consider more complex grammatical forms, Miikkulainen (1996) demonstrated a system that learned the mapping between relative phrase constructions and multiple event representations, based on the use of a stack for maintaining state information during the processing of the next embedded clause in a recursive manner.

In a more generalized approach, Dominey (2000) exploited the regularity that sentence to meaning mapping is encoded in all languages by word order and grammatical marking (bound or free) (Bates et al. 1982). That model was based on the functional neurophysiology of cognitive sequence and language processing and an associated neural network model that has been demonstrated to simulate interesting aspects of infant (Dominey \& Ramus 2000) and adult language processing (Dominey et al. 2003).

\section{Objectives}

The goals of the current study are fourfold: First to test the hypothesis that meaning can be extracted from visual scenes based on the detection of contact and its parameters in an approach similar to but significantly simplified from Siskind (2001); Second to determine whether the model of Dominey (2000) can be extended to handle embedded relative clauses; Third to demonstrate that these two systems can be combined to perform miniature language acquisition; and finally to demonstrate that the combined system can provide insight into the developmental progression in human language acquisition without the necessity of a pre-wired parameterized grammar system (Chomsky 1995).

\section{The Training Data}

The human experimenter enacts and simultaneously narrates visual scenes made up of events that occur between a red cylinder, a green block and a blue semicircle or "moon" on a black matte table surface. A video camera above the surface provides a video image that is processed by a color-based recognition and tracking system (Smart - Panlab, Barcelona Spain) that generates a time ordered sequence of the contacts that occur between objects that is subsequently processed for event analysis (below). The simultaneous narration of the ongoing events is processed by a commercial speech-totext system (IBM ViaVoice ${ }^{\mathrm{TM}}$ ). Speech and vision data were acquired and then processed off-line yielding a data set of matched sentence - scene pairs that were provided as input to the structure mapping model. A total of $\sim 300$ <sentence, scene> pairs were tested in the following experiments.

\section{Visual Scenes and analysis}

For a given video sequence the visual scene analysis generates the corresponding event description in the format event(agent, object, recipient).

\section{Single Event Labeling}

Events are defined in terms of contacts between elements. A contact is defined in terms of the time at which it occurred, the agent, object, and duration of the contact. The agent is determined as the element that had a larger relative velocity towards the other element involved in the contact. Based on these parameters of contact, scene events are recognized as follows:

Touch(agent, object): A single contact, in which (a) the duration of the contact is inferior to touch_duration (1.5 seconds), and (b) the object is not displaced during the duration of the contact.

Push(agent, object): A single contact in which (a) the duration of the contact is superior or equal to touch_duration and inferior to take_duration (5 sec), (b) the object is displaced during the duration of the contact, and (c) the agent and object are not in contact at the end of the event.

Take(agent, object): A single contact in which (a) the duration of contact is superior or equal to take_duration, (b) the object is displaced during the contact, and (c) the agent and object remain in contact.

Take(agent, object, source): Multiple contacts, as the agent takes the object from the source. For the first contact between the agent and the object (a) the duration of contact is superior or equal to take_duration, (b) the object is displaced during the contact, and (c) the agent and object remain in contact. For the optional second contact between the agent and the source (a) the duration of the contact is inferior to take_duration, and (b) the agent and source do not remain in contact. Finally, contact between the object and source is broken during the event. 
Give(agent, object, recipient): In this multiple contact event, the agent first takes the object, and then gives the object to the recipient. For the first contact between the agent and the object (a) the duration of contact is inferior to take_duration, (b) the object is displaced during the contact, and (c) the agent and object do not remain in contact. For the second contact between the object and the recipient (a) the duration of the contact is superior to take_duration, and (b) the object and recipient remain in contact. For the third (optional) contact between the agent and the recipient (a) the duration of the contact is inferior to take_duration and thus the elements do not remain in contact.

These event labeling templates form the basis for a template matching algorithm that labels events based on the contact list, similar to the spanning interval and event logic of Siskind (2001).

\section{Complex "Hierarchical" Events}

The events described above are simple in the sense that there have no hierarchical structure. This imposes serious limitations on the syntactic complexity of the corresponding sentences (Feldman et al. 1996, Miikkulainen 1996). The sentence "The block that pushed the moon was touched by the triangle" illustrates a complex event that exemplifies this issue. The corresponding compound event will be recognized and represented as a pair of temporally successive simple event descriptions, in this case: push(block, moon), and touch(triangle, block). The "block" serves as the link that connects these two simple events in order to form a complex hierarchical event.

\section{Structure mapping for language learning}

The mapping of sentence form onto meaning (Goldberg 1995) takes place at two distinct levels: Words are associated with individual components of event descriptions, and grammatical structure is associated with functional roles within scene events. The first level has been addressed by Siskind (1996), Roy \& Pentland (2000) and Steels (2001) and we treat it here in a relatively simple but effective manner. Our principle interest lies more in the second level of mapping between scene and sentence structure.

\section{Word Meaning}

In the initial learning phases there is no influence of syntactic knowledge and the word-referent associations are stored in the WordToReferent matrix (Eqn 1) by associating every word with every referent in the current scene $(\alpha=0)$, exploiting the cross-situational regularity (Siskind 1996) that a given word will have a higher coincidence with referent to which it refers than with other referents. This initial word learning contributes to learning the mapping between sentence and scene structure (Eqn. 4, $5 \& 6$ below). Then, knowledge of the syntactic structure, encoded in FormToMeaning can be used to identify the appropriate referent (in the SEA) for a given word (in the OCA), corresponding to a non-zero value of $\alpha$ in Eqn. 1. In this "syntactic bootstrapping" for the new word "gugle," for example, syntactic knowledge of Agent-Event-Object structure of the sentence "John pushed the gugle" can be used to assign "gugle" to the object of push.

WordToReferent $(\mathrm{i}, \mathrm{j})=$ WordToReferent $(\mathrm{i}, \mathrm{j})+$
$\operatorname{OCA}(\mathrm{k}, \mathrm{i}) * \operatorname{SEA}(\mathrm{m}, \mathrm{j}) *$
$\alpha$ FormToMeaning $(\mathrm{m}, \mathrm{k})$

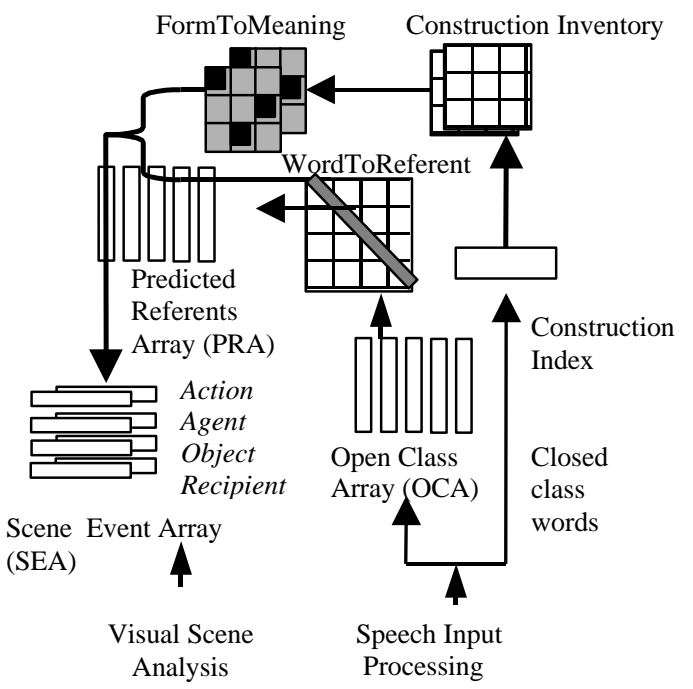

Figure 1. Structure-Mapping Architecture. Open class words in OCA are translated to Predicted Referents in the PRA via the WorldToReferent mapping. PRA elements are mapped onto their roles in the SEA by the FormToMeaning mapping, specific to each sentence type. This mapping is retrieved from Construction Inventory, via the ConstructionIndex that encodes the closed class words that characterize each sentence type.

\section{Open vs Closed Class Word Categories}

Our approach is based on the cross-linguistic observation that open class words (e.g. nouns, verbs, adjectives and adverbs) are assigned to their thematic roles based on word order and/or grammatical function words or morphemes (Bates et al. 1982). Newborn infants are sensitive to the perceptual properties that distinguish these two categories (Shi et al. 1999), and in adults, these categories are processed by dissociable neurophysiological systems (Brown et al. 1999). 
Similarly, artificial neural networks can also learn to make this function/content distinction (Morgan et al. 1996). Thus, for the speech input that is provided to the learning model open and closed class words are directed to separate processing streams that preserve their order and identity, as indicated in Figure 1.

Note that by making this dissociation between open and closed class elements, the grammar learning problem is substantially simplified. Again, it is thus of interest that newborn infants can perform this lexical categorization (Shi et al. 1999), and we have recently demonstrated that a recurrent network of leaky integrator neurons can categorize open and closed class words based on the structure of the F0 component of the speech signal in French and English (Blanc, Dodane \& Dominey 2003).

\section{Mapping Sentence to Meaning}

In terms of the architecture in Figure 2, this mapping can be characterized in the following successive steps. First, words in the Open Class Array are decoded into their corresponding scene referents (via the WordToReferent mapping) to yield the Predicted Referents Array that contains the translated words while preserving their original order from the OCA (Eqn 2).

$$
\operatorname{PRA}(\mathrm{k}, \mathrm{j})=\sum_{\mathrm{i}=1}^{\mathrm{n}} \operatorname{OCA}(\mathrm{k}, \mathrm{i}) * \operatorname{WordToReferent}(\mathrm{i}, \mathrm{j})
$$

Next, each sentence type will correspond to a specific form to meaning mapping between the PRA and the SEA. encoded in the FormToMeaning array. The problem will be to retrieve for each sentence type, the appropriate corresponding FormToMeaning mapping. To solve this problem, we recall that each sentence type will have a unique constellation of closed class words and/or bound morphemes (Bates et al. 1982) that can be coded in a ConstructionIndex (Eqn.3) that forms a unique identifier for each sentence type. Thus, the appropriate FormToMeaning mapping for each sentence type can be indexed in ConstructionInventory by its corresponding ConstructionIndex.

ConstructionIndex $=\mathrm{f}_{\text {circularShift }}($ ConstructionIndex, FunctionWord)

The link between the ConstructionIndex and the corresponding FormToMeaning mapping is established as follows. As each new sentence is processed, we first reconstruct the specific FormToMeaning mapping for that sentence (Eqn 4), by mapping words to referents (in PRA) and referents to scene elements (in SEA). The resulting, FormToMeaningCurrent encodes the correspondence between word order (that is preserved in the PRA Eqn 2) and thematic roles in the SEA. Note that the quality of FormToMeaningCurrent will depend on the quality of acquired word meanings in WordToReferent. Thus, syntactic learning requires a minimum baseline of semantic knowledge.

FormToMeaningCurrent $(\mathrm{m}, \mathrm{k})=$

$$
\sum_{\mathrm{i}=1}^{\mathrm{n}} \operatorname{PRA}(\mathrm{k}, \mathrm{i}) * \operatorname{SEA}(\mathrm{m}, \mathrm{i})
$$

Given the FormToMeaningCurrent mapping for the current sentence, we can now associate it in the ConstructionInventory with the corresponding function word configuration or ConstructionIndex for that sentence, expressed in (Eqn 5).

ConstructionInventory $(\mathrm{i}, \mathrm{j})=$ ConstructionInventory $(\mathrm{i}, \mathrm{j})$

+ ConstructionIndex(i)

* FormToMeaning-Current $(\mathrm{j})$

Finally, once this learning has occurred, for new sentences we can now extract the FormToMeaning mapping from the learned ConstructionInventory by using the ConstructionIndex as an index into this associative memory, illustrated in Eqn. 6.

FormToMeaning $(\mathrm{i})=$

$\sum_{i=1}^{n}$ ConstructionInventory $(\mathrm{i}, \mathrm{j}) *$ ConstructinIndex $(\mathrm{j})$

To accommodate the dual scenes for complex events Eqns. 4-7 are instantiated twice each, to represent the two components of the dual scene. In the case of simple scenes, the second component of the dual scene representation is null.

We evaluate performance by using the WordToReferent and FormToMeaning knowledge to construct for a given input sentence the "predicted scene". That is, the model will construct an internal representation of the scene that should correspond to the input sentence. This is achieved by first converting the Open-Class-Array into its corresponding scene items in the PredictedReferents-Array as specified in Eqn. 2. The referents are then re-ordered into the proper scene representation via application of the FormToMeaning transformation as described in Eqn. 7.

$\operatorname{PSA}(\mathrm{m}, \mathrm{i})=\operatorname{PRA}(\mathrm{k}, \mathrm{i}) *$ FormToMeaning $(\mathrm{m}, \mathrm{k})$

When learning has proceeded correctly, the predicted scene array (PSA) contents should match those of the scene event array (SEA) that is directly derived from input to the model. We then quantify performance error in terms of the number of mismatches between PSA and 
SEA.

\section{Experimental results}

Hirsh-Pasek \& Golinkof (1996) indicate that children can use knowledge of word meaning to acquire a fixed SVO template around 18 months, and then expand this to non-canonical sentence forms around 24+ months. Tomasello (1999) similarly indicates that fixed grammatical constructions will be used initially, and that these will then provide the basis for the development of more generalized constructions (Goldberg 1995). The following experiments attempt to follow this type of developmental progression.

\section{A. Learning of Active Forms for Simple Events}

1. Active: The block pushed the triangle.

2. Dative: The block gave the triangle to the moon.

For this experiment, 17 scene/sentence pairs were generated that employed the 5 different events, and narrations in the active voice, corresponding to the grammatical forms 1 and 2. The model was trained for 32 passes through the $17 \mathrm{scene} / \mathrm{sentence}$ pairs for a total of 544 scene/sentence pairs. During the first 200 scene/sentence pair trials, $\alpha$ in Eqn. 1 was 0 (i.e. no syntactic bootstrapping before syntax is adquired), and thereafter it was 1 . This was necessary in order to avoid the random effect of syntactic knowledge on semantic learning in the initial learning stages. The trained system displayed error free performance for all 17 sentences, and generalization to new sentences that had not previously been tested.

\section{B. Passive forms}

This experiment examined learning active and passive grammatical forms, employing grammatical forms 1-4. Word meanings were used from Experiment A, so only the structural FormToMeaning mappings were learned.

3. Passive: The triangle was pushed by the block.

4. Dative Passive: The moon was given to the triangle by the block.

Seventeen new scene/sentence pairs were generated with active and passive grammatical forms for the narration. Within 3 training passes through the 17 sentences (51 scene/sentence pairs), error free performance was achieved, with confirmation of error free generalization to new untrained sentences of these types. The rapid learning indicates the importance of lexicon in establishing the form to meaning mapping for the grammatical constructions.

\section{Relative forms for Complex Events}

Here we consider complex scenes narrated by sentences with relative clauses. Eleven complex scene/sentence pairs were generated with narration corresponding to the grammatical forms indicated in $5-10$ :

5. The block that pushed the triangle touched the moon.

6. The block pushed the triangle that touched the moon.

7. The block that pushed the triangle was touched by the moon.

8. The block pushed the triangle that was touched the moon.

9. The block that was pushed by the triangle touched the moon.

10. The block was pushed by the triangle that touched the moon.

After presentation of 88 scene/sentence pairs, the model performed without error for these 6 grammatical forms, and displayed error-free generalization to new sentences that had not been used during the training for all six grammatical forms.

\section{Combined Test with and Without Lexicon}

A total of 27 scene/sentence pairs, used in Experiments $\mathrm{B}$ and $\mathrm{C}$, were employed that exercised the ensemble of grammatical forms $1-10$ using the learned WordToReferent mappings. After exposure to 162 scene/sentence pairs the model performed and generalized without error. When this combined test was performed without the pre-learned lexical mappings in WordToReferent, the system failed to converge, illustrating the advantage of following the developmental progression from lexicon to simple to complex grammatical structure. This also illustrates the importance of interaction between syntactic and semantic knowledge that is treated in more detail in Dominey (2000).

\section{E. Some Scaling Issues}

A small lexicon and construction inventory are used to illustrate the system behavior. Based on the independant representation formats, the architecture should scale well. The has now been tested with a larger lexicon, and has learned over 35 grammatical constructions. The system should extend to all languages in which sentence to meaning mapping is encoded by word order and/or grammatical marking (Bates et al. 1982). In the current study, deliberate human event production yielded essentially perfect recognition, though the learning model is relatively robust (Dominey 2000) to elevated scene error rates. 


\section{F. Representing Hierarchical Structure}

The knowledge of the system is expressed in the WorldToReferent and FormToMeaning matrices. In order to deal with complex sentences with embedded clauses, it is necessary to use this same knowledge at different levels of the hierarchy. For this, a "branching mechanism" is necessary, that ordinates the input and output vectors corresponding to meaning and word events. An effective solution to that problem is to learn the branching for each construction as we have done. However, a real account of the human faculty of recursion should be both general (i.e. it should apply to any reasonably complex structure) and plausible (i.e. the branching mechanism should be connectionist). In order to provide this level of generality, neural models need to include a logical "stack" (cf Miikkulainen 1996), in order to process the context of embedded sentences. Complex structures themselves may be represented in a connectionist way, using the Recursive Auto-Associative Memory (Pollack, 1990). In (Voegtlin and Dominey 2003), we proposed a representation system for complex events, that is both generative (it can handle any structure) and systematic (it can generalize, and it does so in a compositional way). This system could be used here, as its representation readily provides a caserole system. The advantages are twofold. First, the branching mechanism is implemented in a neurally realistic way. Second, the recursion capability of the system will allow it to apply its knowledge to any sentence form, whether known or new. Future research will address this issue.

\section{Conclusion}

The current study demonstrates (1) that the perceptual primitive of contact (available to infants at 5 months), can be used to perform event description in a manner that is similar to but significantly simpler than Siskind (2001), (2) that a novel implementation of principles from construction grammar can be used to map sentence form to these meanings together in an integrated system, (3) that relative clauses can be processed in a manner that is similar to, but requires less specific machinery (e.g. no stack) than that in Miikkalanian (1996), and finally (4) that the resulting system displays robust acquisition behavior that reproduces certain observations from developmental studies with very modest "innate" language specificity.

Note that one could have taken the same approach by integrating Siskind's (2001) full event system, and Miikkulainen's (1996) embedded case-role system. Each of these however required significant architectural complexity to accomplish the full job. The current goal was to identify minimal event recognition and form-tomeaning mapping capabilities that could be integrated into a coherent system that performs at the level of a human infant in the first years of development when the construction inventory is being built up. This forms the basis for the infant's subsequent ability to de- and recompose these constructions in a truly compositional manner, a topic of future research.

\section{Acknowledgments}

Supported by the EuroCores OMLL project, the French ACI Integrative and Computational Neuroscience Project, and the HFSP MCILA Project.

\section{Appendix: Sentence and scene descriptions}

The <sentence, meaning> pairs for training and testing are constructed from the following templates. The lexicon consists of 5 nouns (cylinder, moon, block, cat, dog), 5 verbs (touch, push, take, give, say), and 8 function words (to, by, from, was, that, it, itself, and $)^{1}$.

\section{A.1 Single event scenes}

1. Agent verb object. (Active) Verb(agent, object)

2. Object was verbed by agent. (Passive) Verb(agent, object).

3. Agent verbed object to recipient. (Dative) Verb(agent, object, recipient)

4. Object was verbed to recipient by agent. (Dative passive)

Action1(agent1, object2, recipient3).

5. Agent 1 action 1 recipient 3 object 2 .

Verb(agent, object, recipient).

\section{A.2 Double event relatives}

6. Agent1 that verb1ed object2 verb2ed object3. (Relative agent).

Action1(agent1,object2), Action2(agent1,object3)

7. Object 3 was action2ed by agent 1 that action 1 ed object2. (Relative object).

Action1(agent1,object2), Action2(agent1,object3)

8 . Agent 1 that action 21 ed object 2 was action22ed by agent3

Action1(agent1,object2), Action2(agent3,object1)

9. Agent 3 action2ed object 1 that action 1 ed object 2 Action1(agent1,object2), Action2(agent3,object1)

10. Obj2 that was action1ed by agent 1 action2ed obj3 Action1(agent1,object2), Action2(agent2,object3)

11 . Obj 3 was act $2 \mathrm{~d}$ by agent 2 that was act $1 \mathrm{~d}$ by agent 1 Action1(agent1,object2), Action2(agent2,object3)

12 . Obj2 that was action 1 ed by agent 1 was action 2 ed by ag3

Action1(agent1,object2), Action2(agent3,object2)

13. ag 3 act22ed obj 2 that was act21ed by ag 1

Action21(agent1,object2), Action22(agent3,object2)

\footnotetext{
${ }^{1}$ Possible scaling issues for WordToWorld mappings are not of concern here. If WordToWorld is well specified, then lexicon size has no influence on SentenceToWorld mapping.
} 
14. Ag1 that act1ed obj2 act2ed obj3 to recip4 Action1(agent1,object2), Action2(agent1,object3,recipient4)

15. Obj3 was act32ed to recip4 by ag1 that act21ed obj2 Action1(agent1,object2), Action2(agent1,object3,recipient4)

16. Agent 1 that action 1 ed object 2 was action2ed to recip4 by ag3

Action1(agent1,object2),

Action2(agent3,object1, recipient4)

17. Ag3 act2ed obj4 to recip1 that act1ed obj2

Action1(agent1,object2),

Action2(agent3,object4,recipient1)

18. Obj4 was act2ed from ag3 to recip1 that act1ed obj2 Action1(agent1,object2), Action2(agent3,object4,recipient1)

19. Obj2 that was act1ed by ag 1 act2ed obj3 to recip4 Action1(agent1,object2), Action2(agent2,object3,recipient4)

20. Ag3 act2ed ob4 to rec2 that was act1ed by ag1 Action1(agent1,object2), Action2(agent3,object4,recipient2)

21. Ag1 that act1ed obj2 to rec3 act2ed obj4 Action1(agent1,object2,recipient3), Action2(agent1,object4)

22. Obj4 was act2ed by ag 1 that act 1 ed ob 2 to rec3 Action1(agent1,object2,recipient3), Action2(agent1,object4)

23. Ag4 act2ed ob1 that act1ed ob2 to rec3 Action1(agent1,object2,recipient3), Action2(agent4,object1)

24. Ob1 that act1ed ob2 to rec3 was act2ed by ag4 Action1(agent1,object2,recipient3), Action2(agent4,object1)

25. Ag2 that was act1ed by ag1 to rec3 act2ed ob4 Action1(agent1,object2,recipient3), Action2(agent2,object4)

26. Ag4 act2ed obj2 that was act1ed by ag1 to rec3 Action1(agent1,object2,recipient3), Action2(agent4,object2)

\section{A.3 Dual event Conjoined}

27. Agent1 action 1 object1 and object2. (Active conjoined object)

Action1(agent1, object1),

Action1(agent1, object2)

28. Agent 1 and agent 3 action 1 ed object2. (Active conjoined agent)

Action1(agent1, object2), Action1(agent3, object2)

29. Agent 1 action 1 ed object 2 and action 2 object 3 . (Conjoined)

Action1(agent1, object2),

Action2(agent1, object3)

\section{A.4 Dual Event Reflexive}

30. Agent 1 action1r that agent 2 action2ed object3. (Simple reflexive) Action $1 r^{2}$ (agent1), Action2(agent2, object3).

31. Agent1 action1ed itself. (Simple active reflexive) Action1(agent1, agent1).

32. Agent1 action1r that agent2 action2ed itself. (Reflexive simple noun phrase).

Action1r(agent1), Action2(agent2, agent2).

33. Agent 1 action1r that agent 2 action2ed it. (Pronoun simple noun phrase).

Action1r(agent1), Action2(agent2, agent1).

34. Agent1 action1r that it action1ed object2. Action1r(agent1), Action2(agent1, object2).

35 . Agent 1 action1r that object 3 was action2ed by agent 2 . Action1r(agent1), Action2(agent2, object3).

36. Agent 1 action $1 \mathrm{r}$ that agent 2 action 2 ed object 3 to recipient4.

Action1r(agent1),

Action2(agent2, object3, recipient4).

37. Agent 1 action $1 \mathrm{r}$ agent 2 action 2 ed object 3 to recipient4.

Action1r(agent1),

Action2(agent2, object3, recipient4).

38 . Object 2 object 3 were action 1 ed to recipient 4 by agent1.

Action1(agent1, object2, recipient4), Action1(agent1, object3, recipient4)

\section{References}

Bates E, McNew S, MacWhinney B, Devescovi A, Smith S (1982) Functional constraints on sentence processing: A cross linguistic study, Cognition (11) 245-299.

Blanc J-M, Dodane C, Dominey PF (2003) Temporal Processing for Syntax Acquisition: A simulation study. Ms submitted to CogSci 2003.

Brown CM, Hagoort P, ter Keurs M (1999) Electrophysiological signatures of visual lexical processing : Open- and closed-class words. Journal of Cognitive Neuroscience. 11 :3, 261-281

Chomsky N. (1995) The Minimalist Program. MIT

Chang NC, Maia TV (2001) Grounded learning of grammatical constructions, AAAI Spring Symp. On Learning Grounded Representations, Stanford CA.

Dominey PF, Ramus F (2000) Neural network processing of natural lanugage: I. Sensitivity to serial, temporal and abstract structure of language in the infant. Lang. and Cognitive Processes, 15(1) 87-127

\footnotetext{
${ }^{2}$ Corresponds to reflexive verbs such as "said," or "believed."
} 
Dominey PF (2000) Conceptual Grounding in Simulation Studies of Language Acquisition, Evolution of Communication, 4(1), 57-85.

Dominey PF, Hoen M, Lelekov T, Blanc JM (2003) Neurological basis of language in sequential cognition: Evidence from simulation, aphasia and ERP studies, (in press) Brain and Language

Elman J (1990) Finding structure in time. Cognitive Science, 14:179-211.

Feldman JA, Lakoff G, Stolcke A, Weber SH (1990) Miniature language acquisition: A touchstone for cognitive science. In Proceedings of the $12^{\text {th }}$ Ann Conf. Cog. Sci. Soc. 686-693, MIT, Cambridge MA

Feldman J., G. Lakoff, D. Bailey, S. Narayanan, T. Regier, A. Stolcke (1996). L0: The First Five Years. Artificial Intelligence Review, v10 103-129.

Goldberg A (1995) Constructions. U Chicago Press, Chicago and London.

Hirsh-Pasek K, Golinkof RM (1996) The origins of grammar: evidence from early language comprehension. MIT Press, Boston.

Kotovsky L, Baillargeon $\mathrm{R}$, The development of calibration-based reasoning about collision events in young infants. 1998, Cognition, 67, 311-351

Langacker, R. (1991). Foundations of Cognitive Grammar. Practical Applications, Volume 2. Stanford University Press, Stanford.

Mandler J (1999) Preverbal representations and language, in P. Bloom, MA Peterson, L Nadel and MF Garrett (Eds) Language and Space, MIT Press, 365-384

Miikkulainen R (1996) Subsymbolic case-role analysis of sentences with embedded clauses. Cognitive Science, 20:47-73.

Morgan JL, Shi R, Allopenna P (1996) Perceptual bases of rudimentary grammatical categories: Toward a broader conceptualization of bootstrapping, pp 263-286, in Morgan JL, Demuth K (Eds) Signal to syntax, Lawrence Erlbaum, Mahwah NJ, USA.

Pollack JB (1990) Recursive distributed representations. Artificial Intelligence, 46:77-105.

Roy D, Pentland A (2002). Learning Words from Sights and Sounds: A Computational Model. Cognitive Science, 26(1), 113-146.

Shi R., Werker J.F., Morgan J.L. (1999) Newborn infants' sensitivity to perceptual cues to lexical and grammatical words, Cognition, Volume 72, Issue 2, B11-B21.

Siskind JM (1996) A computational study of crosssituational techniques for learning word-to-meaning mappings, Cognition (61) 39-91.

Siskind JM (2001) Grounding the lexical semantics of verbs in visual perception using force dynamics and event logic. Journal of AI Research (15) 31-90

Steels, L. (2001) Language Games for Autonomous Robots. IEEE Intelligent Systems, vol. 16, nr. 5, pp. 1622, New York: IEEE Press.

Stolcke A, Omohundro SM (1994) Inducing probablistic grammars by Bayseian model merging/ In Grammatical
Inference and Applications: Proc. $2^{\text {nd }}$ Intl. Colloq. On Grammatical Inference, Springer Verlag.

Talmy L (1988) Force dynamics in language and cognition. Cognitive Science, 10(2) 117-149.

Tomasello M (1999) The item-based nature of children's early syntactic development, Trends in Cognitive Science, 4(4):156-163

Voegtlin T, Dominey PF (2003) Linear recursive distributed representations (submitted). 